\title{
The Parkes Lecture* Zona pellucida glycoprotein mZP3: a versatile player during mammalian fertilization
}

\author{
Paul M. Wassarman \\ Department of Cell Biology, Mount Sinai School of Medicine, One Gustave L. Levy Place, New York, \\ NY 10029-6574, USA
}

\begin{abstract}
All mammalian eggs are surrounded by a relatively thick extracellular coat, the zona pellucida $(Z P)$, which facilitates fertilization of eggs by a single spermatozoon. The mouse egg ZP is constructed of only three glycoproteins, termed mZP1-3. Each of these glycoproteins consists of a unique polypeptide that is heterogeneously glycosylated with both asparagine-( $\mathrm{N}$-)linked and serine/threonine-(O-)linked oligosaccharides. Polypeptides of $\mathrm{ZP}$ glycoproteins are highly conserved among mammalian species and are similar to polypeptides of egg vitelline envelope glycoproteins of fish, birds and amphibians. One of the mouse $\mathrm{ZP}$ glycoproteins, $\mathrm{mZP} 3$, serves as both a receptor for spermatozoa and an inducer of the acrosome reaction during fertilization. Freeswimming acrosome-intact spermatozoa recognize and bind to certain serine- $\left(\mathrm{O}_{-}\right)$ linked oligosaccharides located close to the carboxy terminus of mZP3 polypeptide and, after binding, undergo the acrosome reaction (cellular exocytosis). In this review, in addition to the background information presented, results of recent experiments using homologous recombination to produce $\mathrm{mZP3}$ null mice and site-directed mutagenesis to inactivate $\mathrm{mZP} 3$ as a sperm receptor and inducer of the acrosome reaction are presented and discussed.
\end{abstract}

\section{Introduction}

For more than two decades, my colleagues and I have carried out research on a single aspect of mammalian fertilization, the species-specific binding of free-swimming spermatozoa to ovulated eggs (Fig. 1). In particular, our research has focused on the identification and characterization of molecules that support this initial step in the fertilization pathway in mice. In this review, I describe one such molecule, a zona pellucida glycoprotein called $\mathrm{mZP} 3$, which performs multiple functions during mammalian fertilization. This glycoprotein is unique to mammalian eggs, from mice to humans, although its ancestors can be found in vitelline envelopes from a wide variety of vertebrate eggs.

\section{Mammalian oogenesis}

Oogenesis in mammals begins early in fetal development and ends, months to years later, in the sexually mature adult (Wassarman and Albertini, 1994). Oogenesis begins with formation of primordial germ cells (PGC) and proceeds through a series of cellular transformations, from PGC to oogonia to oocytes in the fetus, and from oocytes to eggs in

*This review is based on the Parkes Lecture given at the meeting of the Society for the Study of Fertility held at the University of Strathclyde, Glasgow, in July 1998. the adult. Oogonia proliferate by mitosis, whereas oocytes are meiotic cells.

In mice, the germ cell line has extragonadal origins and originates before or during primitive streak formation in embryos of approximately 7 days of age. At approximately 13 days of age, embryos contain a differentiated ovary and migration of PGC is complete; virtually all of the cells have converted to dividing oogonia in the sex cords. As early as day 12 of embryogenesis, a few oogonia enter the preleptotene and then the leptotene stage of the first meiotic prophase; during preleptotene the final round of DNA replication occurs and oogonia become oocytes. It takes approximately 4 days to complete nuclear progression from leptotene to pachytene. By parturition at approximately day 21 , most oocytes have entered late diplotene or the dictyate stage, and by day 5 post partum, nearly all oocytes have reached the dictyate stage, where they remain until they are stimulated to resume meiosis at the time of ovulation. This large pool of small $(12-15 \mu \mathrm{m}$ in diameter) non-growing oocytes is the sole source of unfertilized eggs in the sexually mature adult mammal.

In adult mammals, growth of oocytes is continuous, and ends in either ovulation of a matured oocyte (unfertilized egg) or degeneration of the oocyte and its follicle. Completion of oocyte growth in mice takes 2-3 weeks, during which the diameter of the oocytes increases from 12 to $80 \mu \mathrm{m}$, not including the zona pellucida (ZP); this represents about a 300 -fold increase in volume. Just before 


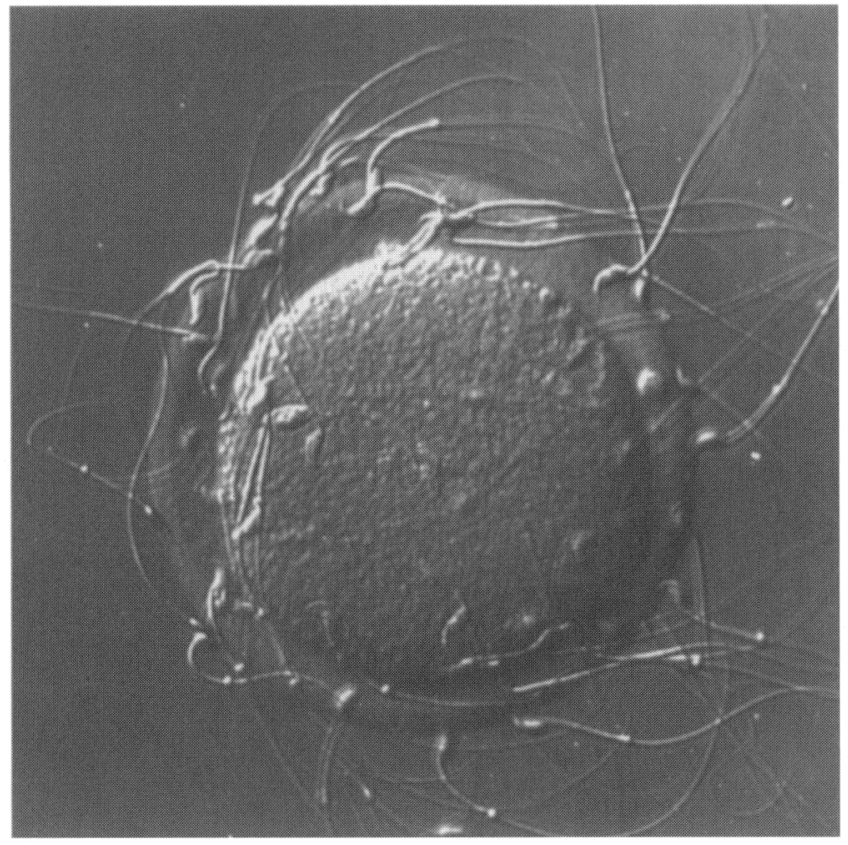

Fig. 1. Photomicrograph of mouse spermatozoa bound to the zona pellucida of an unfertilized mouse egg in vitro.

ovulation, after the preovulatory gonadotrophin (FSH and LH) surge, fully grown oocytes undergo meiotic maturation and become unfertilized eggs arrested at metaphase II of meiosis. The ZP first appears during the earliest stages of oocyte growth, and increases in thickness continually as oocytes increase in diameter.

\section{Mammalian fertilization}

The final steps of mammalian oogenesis and spermatogenesis prepare eggs and spermatozoa, respectively, for fertilization (Gwatkin, 1977; Yanagimachi, 1994; Snell and White, 1996; Wassarman, 1987, 1999). During ovulation, fully grown oocytes from antral follicles undergo meiotic maturation and become unfertilized eggs that are ready to interact with spermatozoa. Similarly, after deposition into, and migration up, the female reproductive tract, spermatozoa undergo capacitation, a process that enables them to bind to eggs and undergo the acrosome reaction (AR). Meiotic maturation of oocytes and capacitation of spermatozoa set gametes off on a path that leads to either formation of a viable zygote or degeneration of the cells.

Typically, very few ovulated eggs are found in the oviducts of females (approximately ten in mice). Similarly, relatively few spermatozoa are found at the site of fertilization (approximately 125 in mice) compared with the number of spermatozoa deposited into the female reproductive tract (approximately 107). A very small percentage of ejaculated spermatozoa reach the position of unfertilized eggs in the oviduct. Whether binding of spermatozoa to eggs occurs due to a chance encounter of gametes in the oviduct or is promoted by a chemical gradient stimulus (sperm chemotaxis), as observed in many nonmammalian species, remains to be resolved conclusively.

Among mammals, the process of the union of germ cells includes several steps that take place in a compulsory order (Fig. 2). It begins in the oviduct with binding of freeswimming spermatozoa to the ovulated egg ZP and ends a short time later $(1-2 \mathrm{~h}$ after combining gametes in vitro) with fusion of egg and sperm plasma membranes to form a single activated cell, the zygote. During this process, several recognizable events take place, including the sperm AR (a form of cellular exocytosis), penetration of the egg ZP by the spermatozoon, and the egg cortical reaction and zona reaction. The latter results in alteration of the $\mathrm{ZP}$ such that free-swimming spermatozoa are unable to bind to fertilized eggs or preimplantation embryos (Fig. 3).

Does mammalian fertilization show any species specificity? It is well known that interspecific hybrids of certain mammals are viable. However, evidence from in vitro fertilization experiments indicates that there are indeed barriers to interspecific fertilization and that the egg ZP serves as a major barrier. The $\mathrm{ZP}$ can interfere with interspecific fertilization by failing to permit the initial binding of spermatozoa to eggs, induction of the AR, or penetration of bound spermatozoa through the extracellular coat. Although the restrictions on binding are not absolute, they provide a relatively high degree of species-specific fertilization in vitro. Notably, removal of the ZP from unfertilized mammalian eggs, thereby exposing egg plasma membrane directly to spermatozoa, eliminates the barrier to interspecific fertilization in vitro for many, but not all mammals (Yanagimachi, 1977, 1981, 1994).

\section{Zona pellucida glycoproteins $\mathrm{mZP1}-3$}

Nearly 20 years ago it was reported that the mouse egg ZP (approximately $6.2 \mu \mathrm{m}$ thick containing approximately $3.5 \mathrm{ng}$ protein) is composed of only three glycoproteins, termed $\mathrm{mZP} 1$ (approximately $200 \mathrm{kDa}$; dimer of identical polypeptides), mZP2 (approximately $120 \mathrm{kDa}$ ), and mZP3 (approximately $83 \mathrm{kDa}$ ) (Bleil and Wassarman, 1980a; Wassarman et al., 1985; Wassarman, 1988a, 1999). Each of these glycoproteins consists of a unique polypeptide ( $\mathrm{mZP1}$, $2 \times$ approximately $75 \mathrm{kDa}$; $\mathrm{mZP} 2$, approximately $75 \mathrm{kDa}$; mZP3, approximately $44 \mathrm{kDa}$ ) that is heterogeneously glycosylated with both complex-type asparagine-( $N$-)linked and serine/threonine- $(O-)$ linked oligosaccharides. As a consequence of the glycosylation, each of the glycoproteins is relatively acidic and appears as a broad band after gel electrophoresis. Each of the polypeptides possesses domains that are conserved among ZP glycoproteins (for example, signal sequences, consensus furin cleavage sites and $\mathrm{ZP}$ domains) (Fig. 4).

It is now apparent that the $\mathrm{ZP}$ of eggs from a wide variety of mammals, including humans, is composed of a small number of glycoproteins that are closely related (polypeptides that have $40-90 \%$ similarity) to mZP1-mZP3. For example, the positions of the cysteine residues, as well as several of the recognizable domains of $\mathrm{mZP} 3$ polypeptide are conserved in human ZP3 polypeptide. Even the vitelline envelope 

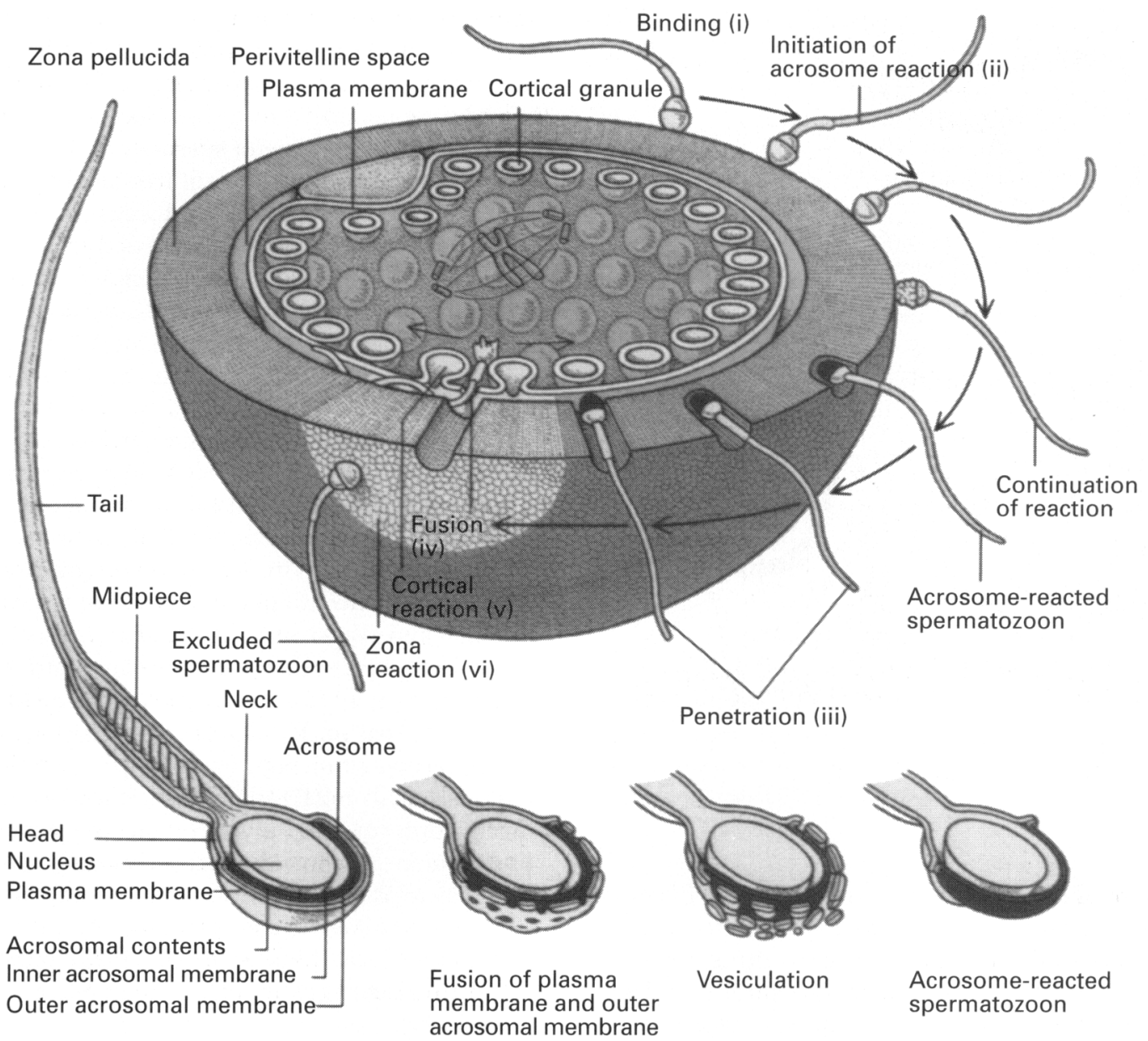

Fig. 2. Schematic diagram of the order of events during fertilization of mammalian eggs. (i) Binding of acrosome-intact spermatozoon to the zona pellucida, (ii) initiation of the acrosome reaction, (iii) penetration of acrosome-reacted spermatozoon through the zona pellucida, (iv) fusion of spermatozoon and egg, (v) the cortical reaction, and (vi) the zona reaction that prevents binding of additional spermatozoa to the zona pellucida. Adapted from Wassarman (1988b).

surrounding eggs from fish, birds, and amphibians contains glycoproteins whose polypeptides resemble mZP1-mZP3. Therefore, there is an evolutionary link between glycoproteins of the vitelline envelope of non-mammalian eggs and glycoproteins of the ZP of mammalian eggs. Since these glycoproteins play essential structural roles in assembly of the extracellular coats during oogenesis (although their synthesis takes place in different sites, for example, in growing oocytes, follicle cells or liver), it is not surprising that they retain common features. It would be of great interest to determine and compare the high resolution structures of vitelline envelope and ZP glycoproteins.

\section{mZP3: a structural glycoprotein}

Glycoproteins of the mouse ZP are organized in a very specific manner. The $Z P$ is composed of long interconnected filaments that are polymers of $\mathrm{mZP} 2$ and $\mathrm{mZP} 3$ (Greve and Wassarman, 1985; Wassarman and Mortillo, 1991). An mZP2-mZP3 dimer is located about every $140 \AA$ along the filaments, thus imposing a structural periodicity that can be

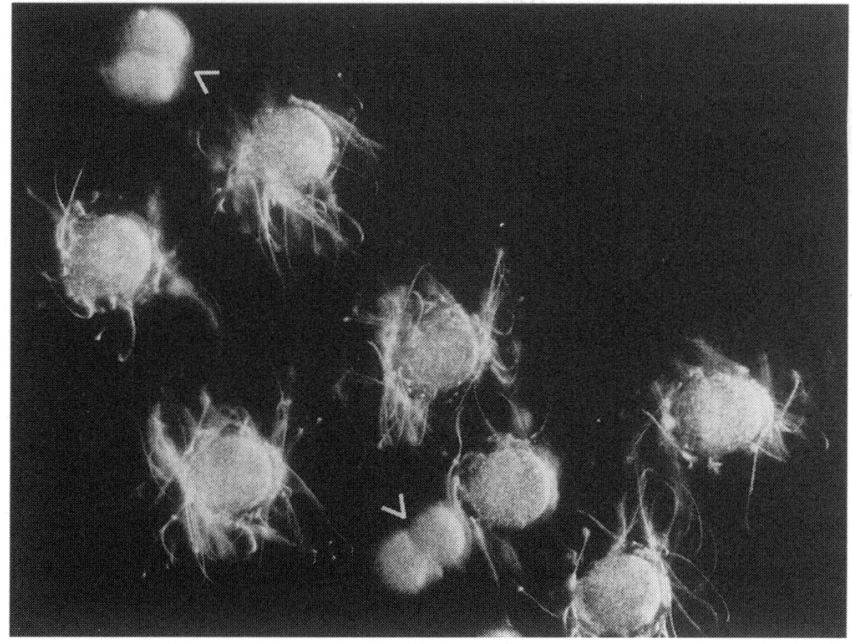

Fig. 3. Light micrograph (dark field) of mouse spermatozoa, eggs and embryos at the end of a competition assay. Spermatozoa are bound to seven ovulated eggs, but are not bound to two two-cell embryos (indicated by arrowheads). 


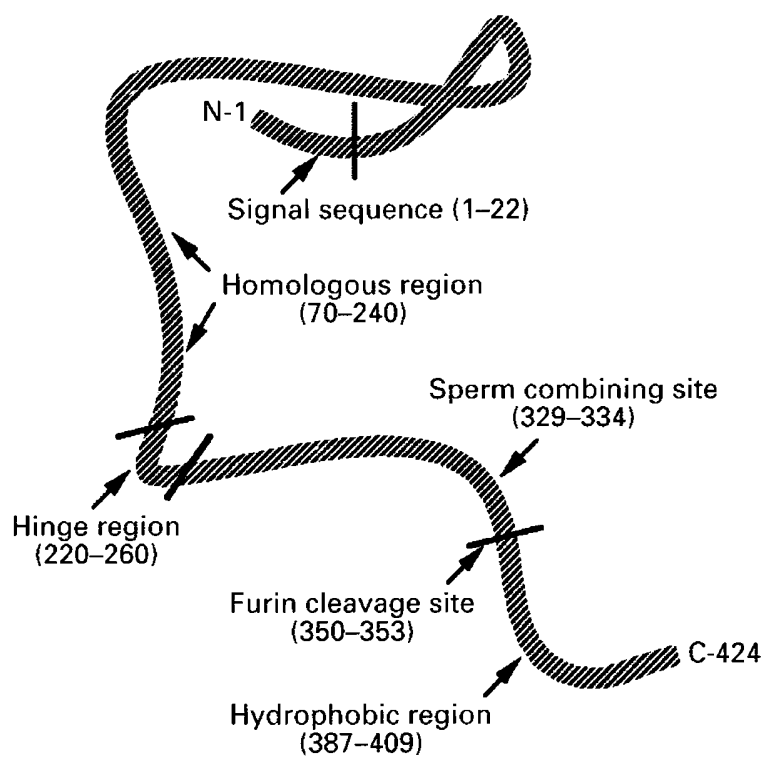

Fig. 4. Schematic diagram of the mZP3 polypeptide showing specific structural and functional domains. It should be noted that the $\mathrm{N}$ terminal signal sequence (amino acids 1-22) and C-terminal fragment (originating at the consensus furin cleavage-site and encoded by exon 8 ) are missing from $\mathrm{mZP} 3$ present in the zona pellucida.

seen in electron micrographs of dissolved ZP. The filaments, in turn, are crosslinked by mZP1 to create a threedimensional matrix. Thus, in addition to other functions, each of the ZP glycoproteins plays a structural role during assembly of the ZP. Recently, it was suggested that assembly of the $\mathrm{ZP}$ from nascent glycoproteins is a stochastic process that may occur completely outside the growing oocyte (Qi and Wassarman, in press).

The mZP3 gene was disrupted by targeted mutagenesis using homologous recombination in mouse embryonic stem cells and null female mice ( $\mathrm{mZP3^{-- }}$ ) were produced to address certain questions about ZP structure and assembly (Liu et al., 1996; Wassarman et al., 1996). When growing oocytes were isolated from ovaries of juvenile $\mathrm{mZP} 3^{--}$mice and unfertilized eggs were recovered from adult $\mathrm{mZP3} 3^{--}$ mice, they were found to lack a ZP. Furthermore, female $\mathrm{mZP3^{-1 }}$ mice were infertile. This observation indicates that in the absence of mZP3 synthesis, a ZP fails to form around growing oocytes during oogenesis, and it emphasizes the critical role played by $\mathrm{mZP} 3$ as a structural glycoprotein.

\section{mZP3: a sperm receptor}

Only acrosome-intact spermatozoa bind to the ovulated mouse egg ZP. Experimental evidence supports the proposal that, during binding of spermatozoa to eggs, $\mathrm{mZP} 3$ serves as a receptor for spermatozoa. For example, of the three glycoproteins that constitute the $\mathrm{ZP}$, only purified mZP3 binds exclusively to the heads of acrosome-intact spermatozoa and thereby prevents them from binding to ovulated eggs in vitro (Bleil and Wassarman, 1980b, 1986; Wassarman, 1990; Mortillo and Wassarman, 1991; Wassarman and Litscher, 1995). Even at nanomolar concentrations, purified unfertilized egg $\mathrm{mZP} 3$ is a very effective inhibitor of sperm binding in this competition assay. However, at similar concentrations, mZP3 from fertilized eggs or early embryos has no effect on binding of spermatozoa to eggs in vitro. This is consistent with the failure of free-swimming spermatozoa to bind to the ZP of fertilized eggs and preimplantation embryos (Fig. 3). It can be concluded from these and other observations (for example, see Bleil and Wassarman, 1980b; Florman and Wassarman, 1985; Wassarman, 1990) that, as a consequence of the zona reaction, $\mathrm{mZP} 3$ is altered such that free-swimming spermatozoa can no longer recognize and bind to the glycoprotein.

The ability of mZP3 to act as a sperm receptor in vitro is not significantly affected by high temperatures, detergents, denaturants or reducing agents, or limited proteolysis. Even after extensive proteolysis of $\mathrm{mZP} 3$, the small glycopeptides produced retain activity as a sperm receptor, although higher than normal concentrations are required (Florman et al., 1983; Florman and Wassarman, 1985). These observations indicate that mZP3 polypeptide does not play a direct role in sperm receptor function. However, there is considerable information to indicate that $\mathrm{mZP} 3$ oligosaccharides play a direct role in sperm receptor function. For example, chemical or enzymic removal of all $\mathrm{mZP} 3$ oligosaccharides results in complete inactivation of the glycoprotein as a sperm receptor. Furthermore, $\mathrm{O}$-linked oligosaccharides recovered from mZP3 by mild alkaline hydrolysis under reducing conditions (Florman and Wassarman, 1985) and certain O-linked related oligosaccharides (Litscher et al., 1995) inhibit binding of spermatozoa to eggs in vitro at micromolar concentrations. Collectively, these observations indicate that species-specific sperm-egg binding in mammals is a carbohydrate-mediated event. However, the identity of sugars on $\mathrm{MZP} 3$ that are recognized by spermatozoa remains an unresolved and controversial issue.

Limited proteolysis (Rosiere and Wassarman, 1992; Litscher and Wassarman, 1996), exon swapping (Kinloch et al., 1995) and site-directed mutagenesis (Kinloch et al., 1995; Chen et al., 1998) have been used to locate essential O-linked oligosaccharides on mZP3 polypeptide. The results of these studies indicate that the essential oligosaccharides are present on just two of five serine residues, Ser-332 and -334, in a region of polypeptide near the C-terminus that is encoded by exon 7 of the mZP3 gene (eight exons) (Fig. 4). Of the five serine residues, only these two are conserved from mouse to human ZP3. Therefore, the numerous amino acid changes neighbouring Ser-332 and -334 that have occurred during evolution may impose changes in the structure of $O$ linked oligosaccharides added to ZP3 and, thereby, affect the species specificity of sperm-egg interactions (Wassarman and Litscher, 1995).

\section{mZP3: an acrosome reaction inducer}

The acrosome is a large secretory vesicle that overlies the nucleus in the apical region of the sperm head (Yanagimachi, 1994). Acrosomal membrane just underlying the plasma membrane is referred to as 'outer' acrosomal membrane and 
that overlying the nucleus is referred to as 'inner'acrosomal membrane. Morphologically, the AR is observed as multiple fusions between outer acrosomal membrane and plasma membrane at the anterior region of the sperm head, extensive formation of hybrid membrane vesicles, and exposure of the inner acrosomal membrane and acrosomal contents. Only acrosome-reacted spermatozoa can penetrate the $\mathrm{ZP}$ and fuse with the plasma membrane of the egg.

There are many different inducers of the $A R$ (Yanagimachi, 1994). However, it is generally accepted that ZP3 is the natural agonist that initiates the AR upon binding of acrosome-intact spermatozoa to the $\mathrm{ZP}$ (Bleil and Wassarman, 1983; Darszon et al., 1996; Wassarman and Florman, 1997; Florman et al., 1998). Criteria are now available that permit the so-called spontaneous AR and the ZP3-induced AR (for example, sensitivity to pertussis toxin) to be distinguished. Although purified $\mathrm{mZP} 3$ and large mZP3 glycopeptides induce spermatozoa to undergo the AR in vitro, small $\mathrm{mZP} 3$ glycopeptides and purified $\mathrm{mZP} 3 \mathrm{O}-$ linked oligosaccharides bind to spermatozoa and inhibit binding to the egg, but do not induce the AR (Wassarman, 1988a, 1990). Leyton and Saling (1989) reported that crosslinking of small mZP3 glycopeptides bound to a spermatozoon can induce the spermatozoon to undergo the acrosome reaction. These findings indicate that induction of the AR by ZP3 may be dependent on multivalent interactions.

\section{Concluding remarks}

There is great diversity in the mechanisms of species-specific fertilization. For example, some non-mammalian eggs lack an extracellular coat (for example nematode eggs), while others have a vitelline envelope and a jelly coat (for example echinoderm and amphibian eggs). In some species, the spermatozoon enters the egg at a particular site (micropyle) without the need for an AR (for example nematodes), whereas in others, spermatozoa undergo the AR upon binding to the jelly coat and then bind in a species-specific manner to the vitelline envelope (for example echinoderms and amphibians). It is possible to consider the $\mathrm{ZP}$, which is unique to all mammalian eggs, as an amalgamation of the jelly coat and the vitelline envelope of some non-mammalian eggs. The ZP is the site of both species-specific sperm receptors and inducers of the AR.

In this paper, the current knowledge of the functions of $Z P$ glycoprotein $\mathrm{mZP} 3$ in fertilization has been discussed. Three main conclusions can be made: (i) $\mathrm{mZP} 3$ is a structural glycoprotein that is required for assembly of the ZP during oogenesis; (ii) $\mathrm{mZP} 3$ is a sperm receptor that supports binding of free-swimming spermatozoa to unfertilized eggs; and (iii) $\mathrm{mZP} 3$ is an inducer of the AR for spermatozoa bound to the ZP of unfertilized eggs.

Although a great deal is known about mZP3, many more questions remain. A pressing issue concerns the nature of the protein associated with the plasma membrane surrounding the sperm head that recognizes and binds to $\mathrm{mZP3}$ oligosaccharides (so-called egg-binding protein). To date, this area of research, although extremely productive, is inconclusive (Wassarman, 1999). A second issue concerns the nature of the ZP3 oligosaccharides that are recognized by egg-binding protein. Very little is known about the structure of these oligosaccharides (for example composition, sequence, and conformation) or their sites on ZP3 polypeptides from different mammalian eggs. Can these oligosaccharides account for the species-specific binding of spermatozoa that is observed experimentally? Finally, the mechanism for inactivation of $\mathrm{mZP} 3$ as a sperm receptor after fertilization remains to be elucidated. It is hoped that answers to these questions will be forthcoming and that such progress will be of some benefit to clinicians involved in the manipulation of human reproduction.

For more than two decades I have collaborated with excellent graduate students, postdoctoral fellows, and research assistants, first at Harvard Medical School (1972-1985), then at the Roche Institute of Molecular Biology (1986-1995), and now at Mount Sinai School of Medicine (1996-present). I am very grateful to these scientists for their many important contributions to our research on mammalian fertilization. Our research was supported in large part by the National Institute of Child Health and Human Development, National Science Foundation, and Hoffman-LaRoche Inc. Finally, I thank Brian Cook and his staff for their extraordinary hospitality during my stay in Glasgow.

\section{References}

Bleil JD and Wassarman PM (1980a) Structure and function of the zona pellucida: identification and characterization of the proteins of the mouse oocyte's zona pellucida Developmental Biology 76 185-202

Bleil JD and Wassarman PM (1980b) Mammalian sperm-egg interaction: identification of a glycoprotein in mouse egg zonae pellucidae possessing receptor activity for sperm Cell 20 873-882

Bleil JD and Wassarman PM (1983) Sperm-egg interactions in the mouse: sequence of events and induction of the acrosome reaction by a zona pellucida glycoprotein Developmental Biology 95 317-324

Bleil JD and Wassarman PM (1986) Autoradiographic visualization of the mouse egg's sperm receptor bound to sperm Journal of Cell Biology 102 1363-1371

Chen J, Litscher ES and Wassarman PM (1998) Inactivation of the mouse sperm receptor, $\mathrm{mZP} 3$, by site-directed mutagenesis of individual serine residues located at the combining-site for sperm Proceedings National Academy of Sciences USA $956193-6197$

Darszon A, Liévano A and Beltran C (1996) Ion channels: key elements in gamete signaling Current Topics in Developmental Biology 34 117-167

Florman HM and Wassarman PM (1985) O-Linked oligosaccharides of mouse egg ZP3 account for its sperm receptor activity Cell $\mathbf{4 1}$ 313-324

Florman HM, Bechtol KD and Wassarman PM (1983) Enzymatic dissection of the functions of the mouse egg's receptor for sperm Devevlopmental Biology $106243-255$

Florman HM, Arnoult C, Kazam IG, Li C and O'Toole CMB (1998) A perspective on the control of mammalian fertilization by egg-activated ion channels in sperm: a tale of two channels Biology of Reproduction 59 12-16

Greve JM and Wassarman PM (1985) Mouse egg extracellular coat is a matrix of interconnected filaments possessing a structural repeat Journal of Molecular Biology 181 253-264

Gwatkin RBL (1977) Fertilization Mechanisms in Man and Mammals Plenum Press, New York

Kinloch RA, Sakai Y and Wassarman PM (1995) Mapping the mouse ZP3 combining-site for sperm by exon swapping and site-directed mutagenesis Proceedings National Academy of Sciences USA 92 263-267

Leyton L and Saling PM (1989) Evidence that aggregation of mouse sperm receptors by ZP3 triggers the acrosome reaction Journal of Cell Biology 108 2163-2168

Litscher ES and Wassarman PM (1996) Characterization of a mouse ZP3derived glycopeptide, gp55, that exhibits sperm receptor and acrosome reaction-inducing activity in vitro. Biochemistry 35 3980-3985 
Litscher ES, Juntunen K, Seppo A, Penttilä L, Niemelä R, Renkonen O and Wassarman PM (1995) Oligosaccharide constructs with defined structures that inhibit binding of mouse sperm to unfertilized eggs in vitro. Biochemistry 34 4662-4669

Liu C, Litscher ES, Mortillo S, Sakai Y, Kinloch RA, Stewart CL and Wassarman PM (1996) Targeted disruption of the $m Z P 3$ gene results in production of eggs lacking a zona pellucida and infertility in female mice Proceedings National Academy of Sciences USA 93 5431-5436

Mortillo S and Wassarman PM (1991) Differential binding of gold-labeled zona pellucida glycoproteins $\mathrm{mZP} 2$ and $\mathrm{mZP} 3$ to mouse sperm membrane compartments Development 113 141-151

Qi $\mathbf{H}$ and Wassarman PM Secretion of zona pellucida glycoprotein mZP2 by growing oocytes from $m \mathrm{ZP}^{+/+}$and $m \mathrm{ZP}^{-/-}$mice Developmental Genetics (in press)

Rosiere TK and Wassarman PM (1992) Identification of a region of mouse zona pellucida glycoprotein $\mathrm{mZP} 3$ that possesses sperm receptor activity Developmental Biology 154 309-317

Snell WJ and White JM (1996) The molecules of mammalian fertilization Cell $85629-637$

Wassarman PM (1987) The biology and chemistry of fertilization Science 235 $553-560$

Wassarman PM (1988a) Zona pellucida glycoproteins Annual Review of Biochemistry 57 415-442

Wassarman PM (1988b) Fertilization in mammals Scientific American 255 78-82
Wassarman PM (1990) Profile of a mammalian sperm receptor Development 108 1-17

Wassarman PM (1999) Mammalian fertilization: molecular aspects of gamete adhesion, exocytosis, and fusion Cell 96 175-183

Wassarman PM and Albertini DF (1994) The mammalian ovum. In The Physiology of Reproduction pp 79-122 Eds E Knobil and JD Neill. Raven Press, New York

Wassarman PM and Florman HM (1997) Cellular mechanisms during mammalian fertilization. In Handbook of Physiology, Section 14: Cell Physiology pp 885-938 Eds JF Hoffman and JD Jamieson. Oxford University Press, New York

Wassarman PM and Litscher ES (1995) Sperm-egg recognition mechanisms in mammals Current Topics in Developmental Biology 30 1-19

Wassarman PM and Mortillo S (1991) Structure of the mouse egg extracellular coat, the zona pellucida International Review of Cytology 130 85-109

Wassarman PM, Bleil JD, Florman HM, Greve JM, Roller RJ, Salzmann GS and Samuels FG (1985) The mouse egg's receptor for sperm: what is it and how does it work? Cold Spring Harbor Symposia on Quantitative Biology 50 11-19

Wassarman PM, Liu C and Litscher ES (1996) Constructing the mammalian egg zona pellucida: some new pieces of an old puzzle Joumal of Cell Science 109 2001-2004

Yanagimachi $\mathbf{R}$ (1994) Mammalian fertilization. In The Physiology of Reproduction pp 189-317 Eds E Knobil and JD Neill. Raven Press, New York 